\title{
Die konservative Revolution: Geistesgeschichtliche Anmerkungen zu Japan
}

\author{
Von Ludger Kühnhardt
}

In einem vor Jahrzehnten erschienenen Buch beschreibt Wilhelm Nestle die Griechen im Ubergang "vom Mythos zum Logos". Zwischen dem 6. und dem 5. vorchristlichen Jahrhundert setzten dort jene schlüsselhaften Bewegungen ein, die in zwei geistesgeschichtlich entscheidenden Bereichen zu großen Veränderungen in der Weltanschauung der Griechen führten: Mit Hesiod begann die Entwicklung zur monotheistisch ausgerichteten Zeus-Verehrung, die das pantheistisch-spiritistische Glaubensgefüge abzulösen antrat und später im Christentum ihre endgültige Aufhebung und Ablösung finden sollte; mit Herodot artikulierte sich ein umwerfend neues Geschichtsbild, welches die Mythologie eines Homer überwand, wenngleich Herodots Entwürfe einem Ethnozentrismus huldigten.

Geschichtsbild und Religion sind Schlüsselelemente einer vom Logos geprägten Weltanschauung. Die Erinnerung an die Erfahrungen der Griechen drängt sich - bei ganz unterschiedlich gelagertem Zusammenhang - bei der Reflexion über die geistesgeschichtliche Stellung Japans auf. Das ostasiatische Land - ein zugegeben gewagter Vergleich - steht erst noch vor dem geistesgeschichtlichen Sprung wie ihn seinerzeit das archaische Griechenland vollzog. Die zweitgrößte Wirtschaftsmacht der modernen Welt als ein dem Mythendenken verhafteter Staat - eine gewiß verwegen anmutende These. Hinter der Kulisse des atemberaubend rasch modernisierten Japan enthüllen sich in der Tat eine Vielzahl von Phänomenen, die aus westlicher Perspektive - und der Okzident ist wohl noch immer mehr als eine die kulturelle Dimension beiseite schiebende Agglomeration von Hochhäusern, Autobahnen, Fernsehgeräten und Supermärkten - das Attribut archaisch rechtfertigen.

\section{Die Mythologie des Geschichtsblicks}

Zum Beispiel der Kalender: Für die Japaner vollzieht sich Geschichte nicht notwendigerweise in einer Abfolge von Ereignissen, deren Sprünge, Verwerfungen und zeitlichen Veränderungen sich doch in einem historisch gebundenen Kontinuum befinden. Der zeitund epochenumschließende Geschichtsbegriff, der das Gewordene aus dem Gewesenen herzuleiten vermag, ist dem japanischen Denken nicht recht zu eigen. Epochen stehen eher abrupt nebeneinander und werden erst durch einen Rückgriff in die nationale Mythologie miteinander verbunden. Geschichtliche Brüche werden umgekehrt nicht als sol- 
che gesehen, sondern als Einheit in verschiedener Ausdrucksform überspielt: Japan is a circle. $^{1}$

Mit der Thronbesteigung Kaiser Hirohitos im Jahre 1926 setzte die Epoche Showa ein, in deren Zahlenfolge seither gerechnet wird. Im Jahr Showa 61 (d. h. 1986) zu leben, heißt nicht nur, nationale Einheit unter dem Tenno zu dokumentieren, sondern auch, das geschichtliche Kontinuum zwischen der Regierungszeit des vorherigen und des derzeitigen Kaisers aufzulösen. Wird der Thronfolger seinen Vater eines Tages beerben, beginnt die Zählweise des japanischen Kalenders wieder im Jahr 1 - in der Diktion der Behörden wie auf dem Titelblatt der Zeitungen.

Jene angedeutete Verzerrung des Geschichtsblicks findet ihre mythologische Úberhöhung in der auch in der seriösen japanischen Historiographie bis heute vertretenen Vorstellung, das japanische Kaiserhaus als die eigentliche Mitte des japanischen Volkes leite seine Abkunft von der Sonnengöttin Amaterasu Omikami ab. ${ }^{2}$ Mehrere Jahrhunderte werden zur historisch tatsächlich belegbaren Geschichte Japans dazugerechnet, um die Kaiserherkunft auf Jimmu Tenno (angeblich 600 v. Chr.) zurückzuführen, einen vermeintlicherweise direkten Abkömmling der Sonnengöttin, in Wahrheit aber eine ebenso mythologische Figur wie Aeneas oder Romulus und Remus. Die Geschichte des japanischen Kaiserreiches - die Taika-Reform des Jahres $645 \mathrm{n}$. Chr. führte unter Úbernahme wesentlicher Strukturelemente der chinesischen Tang-Dynastie zur ersten zentralistisch organisierten und monarchischen Staatsordnung in Japan - ist eindrucksvoll und langwährend genug, um die Bedeutung der Kaiserinstitution für Japan zu erkennen. ${ }^{3}$ Dennoch aber hält sich der mythologische Gedanke an die Abkunft des Volkes und seines Kaiserhauses von der Sonnengöttin Amaterasu Omikami und ihrem angeblich direkten Nachkommen Jimmu Tenno, von dem wiederum sich eine direkte Linie zum derzeitigen Kaiser ziehen lasse.

Auch die Beleuchtung der Rolle Japans während des Zweiten Weltkrieges zeugt von jenem angeführten geschichtlichen Verdrängungsmechanismus. 1982 kam es nach Revisionen japanischer Geschichtsbücher zu einer heftigen diplomatischen Kontroverse mit der Volksrepublik China und anderen von Japan während der dreißiger und vierziger Jahre überfallenen und besetzten Staaten der Region. Die Chinesen sprachen von einer Verharmlosung des Kriegsgeschehens, während die Japaner ihre Aktionen auf dem Fest-

1 Zu den Grundströmungen des Denkens in Japan: Moore, Charles A. (Hrsg.), The Japanese Mind. Essentials of Japanese Philosophy and Culture, Tokio/Rutland (Vermont) 1982 (5. Auflage); Schinzinger, Robert, Japanisches Denken, Berlin 1983.

2 Die beiden ältesten Zeugnisse der klassischen japanischen Literatur und Mythenbeschreibung sind: Nihongi. Chronicles of Japan from the earliest times to A.D. 697. Translated from the original Chinese and Japanese by W.G. Aston, Tokio/Rutland (Vermont) 1982 (6. Auflage); The Kojiki. Records of ancient matters. Translated by Basil Hall Chamberlain, Tokio/Rutland (Vermont) 1982; fairerweise bedarf es sogleich an dieser Stelle des Hinweises darauf, daß der Kaiser seit 1945 nurmehr das repräsentative Oberhaupt des japanischen Staates ist und im Zuge der Verf assungsreform am Ende des Zweiten Weltkrieges seiner gottähnlichen Rolle abschwören mußte, vgl.: Mosley, Leonard, Ein Gott- tritt ab. Hirohito - Kaiser von Japan, Oldenburg/Hamburg 1967.

3 Vgl. Ishii, Ryosuke, A history of political institutions in Japan, Tokio 1980, Seite $17 \mathrm{ff}$. 
land als schlichtes Vordringen, als quasi-naturgegebenes Phänomen herunterzuspielen versuchten; die Chinesen verwiesen etwa auf das barbarische Verbrechen an mehr als 20000 Zivilisten in Nanking, von dem die japanischen Schulbuchtexte nichts wissen wollten, während die Japaner eine Kriegsschuld auf allen Seiten und Ubergriffe auf Zivilisation als natürlichen Preis jedweder Kriegsführung zu relativieren suchten. ${ }^{4}$

Besondere Aufmerksamkeit in diesem Zusammenhang fand in Japan der fünfstündige Filmstreifen "Tokyo Saibany (Tokio Tribunal). Der Dokumentarfilm über den japanischen Kriegsverbrecherproze $ß$ - allerorten in Japan 1983/84 ein Kassenschlager zumal unter Jugendlichen - endet mit der apologetischen und relativistischen Aufzählung aller nach 1945 ausgebrochenen Kriege und dem berühmten Photo napalmgeschädigter, schreiender Kinder während des Vietnam-Krieges; da Viele Kriegsschuld auf sich geladen haben, so der suggestive Unterton, können General Tojo und die anderen japanischen Kriegsverantwortlichen nicht schlimmer gewesen sein. ${ }^{5}$

Das zentrale Problem in der japanischen Diskussion um den Zweiten Weltkrieg ist die Vorgeschichte des Pazifischen Krieges, nicht so sehr der Kriegsverlauf selbst. Die Fakten sind eindeutig. 1931 eroberte Japan die Mandschurei. 1932 endete mit der Ermordung von Premierminister Inukai die Ära der Parteienkabinette in Tokio und mußte dem Aufstieg militärischer und militaristisch-nationalistischer Kreise Platz machen. Im selben Jahr trat Japan aus dem Völkerbund aus. 1936 wurde mit dem Deutschen Reich der Antikomintern-Pakt geschlossen, dem sich 1937 Italien anschloß. Im Juli 1937 brach der Krieg mit China nach dem Zwischenfall an der Marco-Polo-Brücke nahe Peking endgültig aus: die Mandschurei wurde als Kaiserreich Manschukuo japanischer Satrapenstaat. Nach der französischen Niederlage in Europa besetzte Japan 1940 Französisch-Indochina. Im April 1941 unterzeichnete Tokio ein Neutralitätsabkommen mit der Sowjetunion, um den Rücken bei der Expansion nach Südostasien und in den Pazifischen Raum frei zu haben. Am 7. Dezember 1941 schließlich wurde der amerikanische Flottenstützpunkt Pearl Harbour angegriffen. Die pazifische Komponente des Zweiten Weltkrieges strebte ihrem Höhepunkt zu. ${ }^{6}$

Die Vereinigten Staaten, Hauptgegner des imperialen Japan, sahen die Hauptursache

4 Vgl. Krebs, Gerhard, Tendenzen der japanischen Zeitgeschichtsschreibung, Tokio 1983; zu einem hervorstechenden Aspekt jener Fragestellung: Jansen, Marius, Japan and China. From war to peace 1894-1972 Chicago 1975; zur historischen Visite des chinesischen kommunistischen Parteivorsitzenden in Tokio, bei welcher Gelegenheit ein "Sino-Japanese Friendship Committee for the 21 st Century" initiiert wurde, aus chinesischer Sicht: A historic visit to Japan. Speeches of Hu Yaobang, General Secretary of the CPC Central Committee (November 23-30, 1983), Peking 1983. Lautes Aufschreien rief der ehemalige US-Botschafter und weltbekannte Japanologe Edwin O. Reischauer in der japanischen Offentlichkeit hervor, als er Ende 1983 den Atombombenabwurf im August 1945 in Hiroshima und Nagasaki als eine zwingende politische Notwendigkeit bezeichnete, um die japanische Kriegsführung zur Kapitulation zu zwingen und damit noch weit größeres Blutvergießen zu verhindern, welches die Konsequenz einer schrittweisen Invasion der amerikanischen - und möglicherweise auch der sowjetischen Truppen - gewesen wäre.

5 Vgl. Murakami, Hyoe, Japan. The years of trial 1919-52, Tokio 1983; Minear, Richard H., Victors' justice. The Tokyo War Crimes Trial, Tokio/Rutland (Vermont) 1982 (2. Auflage).

6 Vgl. Crowley, James C., Japan's quest for autonomy. National security and foreign policy 1930-1938, Princeton 1966. 
des japanischen Expansionismus im autoritären und feudalen Charakter des Kaiserreiches. Entsprechend wurden nach den Atombombenabwürfen, der japanischen Kapitulation und der amerikanischen Okkupation des Inselreiches die Schwerpunkte jener Umwälzung gesetzt, denen Japan unterzogen wurde, um einen demokratischen und freiheitlichen Neuanfang zu ermöglichen. Eine Bodenreform wurde durchgeführt, politische Parteien wurden neubegründet, freie Gewerkschaften und eine freie Presse installiert; die Verfassungsänderung unter dem Einfluß des amerikanischen Oberbefehlshabers General Douglas MacArthur beseitigte den gottähnlichen Status des Kaisers und beließ ihm nurmehr die von den Amerikanern durchaus gewünschte Rolle eines repräsentativen Staatsoberhaupts. $^{7}$

\section{Shintoismus - die große Reinigung}

Eine entscheidende Wurzel für jenen Geschichtsblick, in dem sich historische Fakten mit Mythen und Verdrängungen mischen, findet sich im japanischen Kult des Shintoismus. ${ }^{8}$ Das Böse, das Úbel gilt als Eindringling in den prinzipiell guten Menschen. "O-harai", die große Reinigung, wird als erlösender, die Vergangenheit im Selbstvollzug bereinigenden und sie aufhebenden Akt verstanden. "O-harai" wird an den großen Shinto-Festen vor Tempelanlagen im ganzen Land praktiziert und gilt als symbolische Anrufung der "kami", der Gottheiten, um durch sie Gnade und Reinigung zu erfahren.

Der Gedanke der Reinigung - ins westliche, vom Christentum geprägte Verständnis übertragen: der Erlösung von aller Schuld, ohne daß hingegen im christlichen Verständnis die Erbsünde eliminiert werden könnte, die der Shintoismus nicht kennt - erleichtert den Japanern den Umgang mit sich selbst und ihrer Vergangenheit. Der ethische Pragmatismus resultiert aus diesem Shinto-Weltbild.

Zum Jahresende ziehen die Belegschaften vieler Firmen zu einem Betriebsfest, das quasi als Reinigung von allen im Verlauf des verflossenen Jahres unter den Kollegen angestauten Aversionen und Ressentiments verstanden wird. Im neuen Jahr kann wieder von vorne angefangen werden - ohne die Bürde nachwirkender Vergangenheit, aber auch ohne die Verwurzelung in ihr. Ähnlich vollzieht sich in Japan die politische Auseinandersetzung mit dem Gewesenen. Man lebt im Jetzt. Dennoch aber wirkt die eigene Geschichte nach, holt jedes Volk ein.

Natürlich existieren auch in Japan die Kräfte und Vertreter des Logos und der Rationalität. Allerdings fließen auf verwegene Weise Elemente des Mythos und des Logos zusammen, die dem kartesianisch denkender Abendländer wie Feuer und Wasser erscheinen wollen. Das Weltbild der Japaner aber scheint zur Vereinigung von Gegensätzlichkeiten fähig zu sein, die die einen als Assimilation unterschiedlicher Geistesströmungen, 
die anderen als unreflektiertes Aufsaugen und Imitieren, als pragmatische Wertindifferenz kritisieren.

Verwirrend an Japan ist vieles und zuweilen hat es den Anschein, als würden die Japaner sich hinter der Wand ihrer kulturellen Homogeneität zu verschanzen versuchen. ${ }^{9}$ Die rasanten und tiefgreifenden ökonomischen Erfolge des fernöstlichen Inselstaates nötigen dem Westen Respekt ab und zwangen ihm eine machtvolle Konkurrenz auf den Weltmärkten auf. Geistesgeschichtlich aber ist Japan noch immer ein weithin unentdecktes Land geblieben, wobei die Verantwortung dafür nicht allein bei den allzu einseitig ökonomisch interessierten Europäern und Amerikanern liegt, sondern auch daran, daß die Japaner es auf geschickte Weise verstehen, unter sich zu bleiben, und ausländische Interpreten ihrer Geistestraditionen, Kulturerscheinungen und Lebensweise auf Distanz halten. "Das Gesicht hinter einer Maske verbergen", so beschrieb der 1970 durch klassischen Harakiri-Selbstmord aus dem Leben geschiedene nationalistische Schriftsteller Yukio Mishima das Ideal der japanischen Mentalität.

Dabei gilt es gerade die geistigen und historischen Antriebskräfte zu erfassen, um einen wichtigen Blickwinkel auf dieses faszinierende, wichtige und widersprüchliche Land am Ostrand Asiens freizulegen. Im Gegensatz zu vielen anderen, von Europäern kolonialisierten Gebieten der Erde ist Japan nicht das Produkt abendländischer Zivilisations- und Kolonialisierungsbestrebungen, sondern Ausdruck seiner eigenen Stärke und kulturellen Eigentümlichkeiten.

\section{Japans geschichtlicher Weg}

Die Modernisierung Japans gehört zu den interessantesten und wichtigsten welthistorischen Prozessen des 19. Jahrhunderts. ${ }^{10}$ Während weite Teile der Welt vom europäischen Imperialismus und Kolonialismus erfaßt wurden, konnte Japan eine politische Eigenständigkeit und Unabhängigkeit bewahren, mit deren Hilfe das Land zu einer regional- und weltpolitischen Größe aufzusteigen vermochte. Während viele der heutigen Entwicklungsländer nach dem Abzug der ehemaligen Kolonialmächte - deren Erbmasse oft "westlicher" anmutet als dieses in Japan der Fall ist - vor fast unlösbar erscheinenden

9 Vgl. die nicht ironielosen und bissigen Darstellungen zweier japanischer Autoren, die keinen Anspruch auf wissenschaftliche Solidität erheben, dennoch aber gerade in ihrem essayistisch erf rischenden Stil erhellend für wesentliche Aspekte der japanischen Gesellschaft und ihrer Traditionen sind: Kawasaki, Ichiro, Japan unmasked, Tokio/Rutland (Vermont) 1982 (19. Auflage); Ben-Dasan, Isaiah, The Japanese and the Jews, New York/Tokio 1983 (3. Auflage).

10 Aus der umfangreichen Literatur vgl.: Reischauer, Edwin O./Craig, Albert M., Japan: Tradition and transformation, Tokio/Rutland (Vermont) 1981 (3. Auflage); Bendix, Reinhard, Könige oder Volk. Machtausübung und Herrschaftsmandat, Frankfurt 1980, Seite $288 \mathrm{ff}$.; Norman, E.H., Origins of the modern Japanese state, New York 1975; Maruyama, Masao, Thought and behaviour in modern Japanese politics, London 1963; Ward, Robert E. (Hrsg.), Political development in modern Japan, Princeton 1968; Hall, John W./Jansen, Marius, Studies in the institutional history of early modern Japan, Princeton 1968; Duus, Peter, The rise of modern Japan, Boston 1976. 
Problemen stehen, scheint Japan auch besser als viele westliche Industrieländer für die Bewältigung wesentlicher technologisch-ökonomischer Herausforderungen des aufziehenden 21. Jahrhunderts gerüstet zu sein.

Am Anfang dieser Entwicklung stand eine dezidiert eigenwillige und eigenständige Geschichte, die als die Genese des modernen Japan unabdingbare Wurzeln zu legen vermochte. Die japanische Geschichte läßt sich verkürzend in einige klar geschiedene Epochen unterteilen. ${ }^{11}$ Die Regentschaft des Prinzen Shotoku Taishi (593-622 n. Chr.) gilt als der Beginn der historisch belegbaren einheitlichen Regierung über den größten Teil des Inselreiches und mithin als Ausgangspunkt der dynastischen Tradition Japans. Die Nara-Zeit (710-784) erlebte die Úbernahme entscheidender Elemente der Staatsführung aus dem China der Tang-Dynastie und die Errichtung der ersten japanischen Hauptstadt in Nara. Von 784 bis 1185 (Heian-Zeit) wurde der Regierungssitz nach Kyoto verlegt. Japan begann, sich aus sich selbst heraus weiterzuentwickeln.

Dieses aber gelang nicht nur friedlich. Kriege zwischen den führenden Familienclans der Minamoto und Taira (Gempei-Krieg) endeten in der Zerrüttung des Landes und der schließlichen Errichtung einer Militärregentschaft in Kamakura (1185-1333). In diese Zeit fällt auch die Verleihung des Titels "Shogun" (Großfeldmarschall) an Yoritomo durch den Kaiser. Das in Kamakura angesiedelte Bakufu, die Militärregierung, übernahm die faktische Macht, doch blieb der Kaiser nominelles Staatsoberhaupt.

Als dunkle Epochen der japanischen Geschichte gelten die Ashikaga-Zeit (1338-1573) und die Azuchi-Momoyama-Zeit (1568-1600); der interne Zerrüttungsprozeß des Landes in Form permanenter Feudalfehden wurde überschattet von der Ankunft der ersten Ausländer (portugiesische Händler 1542 oder 1543 in Tanegashima, der Missionar Fraciscus Xavier 1549), der Einführung von Feuerwaffen und des Christentums, das sich sogleich einer vehementen Bekämpfung ausgesetzt sah. ${ }^{12}$

Oda Nobunaga, Toyotomi Hideyoshi und Tokugawa Ieyasu hießen die starken Männer am Ende dieser internen Zerrüttung Japans. Sie gaben der Verwaltung des Landes neue Konturen (Einführung eines nationalen Katasters 1595), versuchten sich expansionistisch (Hideyoshis Invasionsversuch in Korea 1592-1598 scheiterte am Ende) und gerieten schließlich untereinander in Konflikt.

Sieger der Schlacht von Sekigahara 1600 war Tokugawa Ieyasu, der 1603 den Titel "Shogun" erwarb. ${ }^{13}$ Mit den verschiedenen Zweigen seiner Familie errichtete er die Tokugawa-Herrschaft, die bis 1868 währte. Diese lange Epoche japanischer Feudalgeschichte erlebte die Einigung des Landes, den landwirtschaftlichen und kulturellen Aufschwung, den weiteren Ausbau der Bürokratie, aber auch die größten und blutigsten

11 Zur japanischen Geschichte insgesamt: Hall, John Whitney, Japan. From prehistory to modern times, Tokio/Rutland (Vermont) 1983 (12. Auflage); Mason, R.H.P./Caiger, J.G., A history of Japan, Tokio/Rutland (Vermont) 1983 (9. Auflage); Dettmer, Hans A., Grundzüge der Geschichte Japans, Darmstadt 1973.

12 Literarisch hat diese dunkle Epoche ein würdiges und zugleich historisch erhellendes Denkmal in dem Roman "Chinmoku" (Silence) des renommierten katholischen Novelisten Shusako Endo gef unden.

13 Vgl. Sadler, A.L., The maker of modern Japan. The life of Shogun Tokugawa, Ieyasu, Tokio/Rutland (Vermont) 1981 (4. Auflage). 
Christenverfolgungen - vor allem diejenigen von 1622/23 - und die Abschließungsorder "Sakoku-rei" von 1639, die den Außenhandel auf die Holländer begrenzte, die in Deshima (Nagasaki) angesiedelt wurden; ansonsten wurde Japan weithin für Ausländer gesperrt.

Die inneren Reformen der Tokugawa-Zeit legten den Grundstein für jene prämoderne Figuration, die als Basis des weiteren Entwicklungs- und Modernisierungsweges Japans dienen sollte. Der innere Auszehrungsprozeß der polizeistaatlich herrschenden Tokugawa-Familie - der Kaiser wurde als noch immer nominelles Staatsoberhaupt in seinem Palast in Kyoto gehalten - wurde durch ausländischen Druck auf Japan in der Mitte des 19. Jahrhunderts nurmehr unübersehbar.

Es wäre zu einfach, das Ende des Tokugawa-Shogunats und den Einbruch der Moderne einzig auf die Ankunft der schwarzen Schiffe des amerikanischen Commodore Matthew Perry 1854 vor Uraga zu datieren und den zwangsweisen Aufbruch der japanischen Selbstisolation als den einzigen Motor für die folgenden inneren Reformen anzusehen. In Wirklichkeit überwiegen die innenpolitischen und ökonomischen, aber auch geistigpolitischen Entwicklungen beim Zustandekommen des Tokugawa-Zusammenbruchs und der anschließenden Meiji-Restauration.

Wie im 7. Jahrhundert, d. h. vor der Taika-Reform, die zur Ubernahme wesentlicher Elemente des chinesischen Systems jener Zeit führte, so gab auch in der Mitte des 19. Jahrhunderts die innere Instabilität entscheidenden Anlaß zu Veränderungen, um das japanische System durch Reformen vor einer fremden Kolonialisierung zu bewahren. ${ }^{14}$ Der wirtschaftliche Niedergang jener Zeit traf vor allem die unteren Gruppierungen der Kriegerkaste, der Samurai, die nicht länger von der Herrschaft des Tokugawa und der ihm ergebenen Provinzfürsten, der Daimyo, profitieren konnten und aufgrund überhoher Angaben finanziell ausgeblutet waren. Der Keim für eine Rebellion innerhalb der Feudalordnung war gelegt, wie sie vor allem von den beiden südlichen Regionen Satsuma und Choshu ausgehen sollte. Daneben stand eine stark zunehmende geistige Bewegung, die sich vor allem in der historischen Schule von Mito Fragen einer notwendigen inneren Reform des Landes zuwandte.

Von 1824 bis 1832 waren Mißernten weit verbreitet. Eine ernste Hungersnot suchte Nordjapan 1823 heim; 1836 kam es zu einer landesweiten Hungerkatastrophe. Ab Mitte der dreißiger Jahre des 19. Jahrhunderts sahen die Städte sich großen Scharen entwurzelter Bauern gegenüber, die Handarbeit suchten. Gewaltausbrüche und Plünderungen von Reislagern nahmen zu. Hilfsaktionen der Behörden blieben ergebnislos. Die unteren Samurai verarmten immer rascher, ihre Verschuldung wurde beängstigender. Die zunehmende Not der Landbevölkerung wurde von den unzufriedenen Daimyo, den Provinzfürsten, aufgegriffen und militärisch gegen das Tokugawa-Shogunat gewendet. Im Lichte dieser inneren Entwicklungen steht die Ankunft ausländischer Schiffe vor der

$14 \mathrm{Vgl.} \mathrm{zu}$ den divergierenden Strömungen, die sich in Japan zu dieser Zeit artikulierten: Jansen, Marius (Hrsg.), Changing Japanese attitudes toward modernization, Princeton 1965. 
japanischen Küste - der Russe Rezanov war schon 1804 in Nagasaki eingetroffen und hatte vergeblich versucht, für sein Land Handelskonzessionen zu erzwingen -, die die Offnung Japans für den Uberseehandel zum Ziel hatten. Die Symbolkraft jener Schiffe 1856 wurde Japan dann zur Unterzeichnung ungleicher Verträge gezwungen - ist nicht zu übersehen. Dennoch aber war die Tokugawa-Zeit keineswegs ausschließlich eine Epoche der nationalen Isolation und Abschottung. Handelskontakte nach China und Korea waren nach 1639 auf rechterhalten worden, über die Holländer gelangten Informationen zum Weltgeschehen und über die Neuerungen in Europa ins Land und in Japan selbst wurde die geistige Entwicklung des Wesens selektiv, aber durchaus rezipiert: 1720 war vom Shogun Yoshimune der Importbann für ausländische Bücher aufgehoben worden; 1745 gab Aoki Konyo ein erstes holländisch-japanisches Wörterbuch heraus; 1774 übersetzte Sugita Gempaku die "Tafel Anatomia"; Rangaku, holländische Studien führten unter anderen zur Einführung westlicher medizinischer Techniken und staatspolitischer Termini. 1811 dann wurde ein Ubersetzungsbüro eingerichtet, das 1856 als "Institut for the Study of Barbarian Books" eine Namenswandlung erfuhr (Bansho shirabesho) und 1862 als "Institut for Development" weitergeführt wurde, ehe es 1877 in die neugegründete Tokio Universität einging.

\section{Meiji-Isshin: Die konservative Revolution}

Die Fakten jener Epoche, die im Japanischen als "Meiji-Isshin", d. h. eigentlich als Meiji-Revitalisierung, bezeichnet, im westlichen Sprachduktus aber wechselweise als MeijiRevolution oder Meiji-Restauration zu klassifizieren gesucht werden, sind in ihrem politischen Gehalt rasch erzählt: 1867 war der nur vierzehnjährige Mutsuhito als Kaiser Meiji auf den Kaiserthron gestiegen; im gleichen Jahr übernahm mit Hitotsubashi Yoshinobu der 15. und letzte Tokugawa-Shogun sein Amt; schon im November diesen Jahres aber mußte er zurücktreten. Am 3. Januar 1868 besetzten Soldaten des Satsuma-Regionalfürsten den Tokugawa-Palast in Edo, dem heutigen Tokio, und verkündeten die Wiedereinsetzung des Kaisers als des nicht nur nominellen, sondern auch faktischen Herrschers im Lande. Der Landbesitz des Shoguns und jener ihn tragender Daimyos wurde konfisziert; bis Mai 1869 regte sich noch auf der Nordinsel Hokkaido militärischer Widerstand der Shogun-Armee.

Trotz der Unruhen unter den Bauern war die Meiji-Restauration (osei-fukko) - und dieser Begriff hat sich in der Literatur weithin eingebürgert - weder eine Bauern - noch eine Bürgerrevolte. Die Anführer des Wandels vom Militärregime des Shogunats zur Kaiserherrschaft fanden sich vor allem unter der Kriegerkaste, den Samurai. Unter dem Eindruck der anwachsenden ausländischen Bedrohung und dem inneren Zerfallsprozeß im Lande selbst ging es den Trägern der Meiji-Revolte in erster Linie um eine Abwehr der "Barbaren" durch die Stärkung und Neubelebung der alten imperialen Traditionen Japans. Die Meiji-Restauration war ein "counterrevolutionary drive toward political ab- 
solutism ". ${ }^{15}$ Ohne eigentlich eine Revolution durchgeführt zu haben, hatten die Japaner die politischen Machtverhältnisse in ihrem Lande grundlegend verändert.

Der Kurs des neuorientierten Staatswesens aber mußte sich erst in den kommenden Jahren und Jahrzehnten herauskristallisieren. Der Westen stand als Hoffnung auf Fortschritt und Stärke vor den Intellektuellen Japans, ohne daß sie hingegen "westlich" zu werden gedachten. ${ }^{16}$ Unter der Parole "bummei kaika" (Zivilisation und Aufklärung) richtete sich der Blick der japanischen Modernisierer jener Generation - so etwa Fukuzawa Yukichis - auf die technologische Infrastruktur des Westens, auf Einsenbahnen, Banken, Museen, das Militär und die Universitäten; die geistige Basis des Abendlandes, vor allem auch das Christentum, sollte hingegen keinen Einzug in Japan halten.

Schon in den vierziger Jahren des 19. Jahrhunderts hatten Intellektuelle nach dem Westen geschaut und dazu aufgerufen, daß eine militärische Ausrüstung mit westlichen Waffen für Japan notwendig sei. Sie - darunter vor allem Sakuma Shozan (1811-1864) und Takano Choei (1804-1850) und die Gruppe der historischen Mito-Schule - propagierten die Neubelebung des "kokutai«, des nationalen Körpers aus Staat und ShintoKult, mit dem Kaiser als gottähnlichem Oberhaupt an der Spitze.

Von einer einheitlichen Zielperspektive der Meiji-Reformer kann nicht gesprochen werden. Das intellektuelle Klima Japans in jenen Jahren bis zur Verabschiedung der eher neokonfuzianisch geprägten Meiji-Verfassung im Jahre 1889 wurde von unterschiedlichen Tendenzen erfaßt und bestimmt. Der Schlachtruf der Anhänger einer imperialen Restauration lautete "sonno joi" (Verehrt den Kaiser, weist die Barbaren zurück). Dieses Zentralmotto der Verfechter einer Reinthronisation des bisher nur nominell herrschenden Kaisers als der obersten Staatsgewalt appellierte sowohl an die gegen die Militärherrschaft des Shogunats und seiner Regierung, des Bakufu, orientierten Gefühle als auch gegen die westlichen Ausländer und ihre an Japan gerichteten Forderungen nach Offnung des Landes für den Handel. Ihr eigentliches Ziel orientierte sich an einer nationalistisch-imperialen Selbststärkung unter Zuwendung zur shintoistischen Kulttradition.

Auf der anderen Seite standen die mit ihrem Programm nach vorne gerichteten Aufklärer, deren Motto "bummei kaika" (Zivilisation und Aufklärung) ihre Zielperspektive populistisch zusammenfaßte. Im Gegensatz zu den ausländerfeindlichen Soupçons spielten diese eher liberalen Kräfte mit dem Gedanken, neben westlicher Waffentechnologie auch demokratische und verfassungsstaatliche Elemente aus dem Westen zu übernehmen. ${ }^{17}$

15 Hall, John Whitney, Japan: From prehistory to modern times, a.a.O., Seite 267.

16 In einem größeren historischen Zusammenhang zur Frage der Berührung der japanischen mit der westlichen Welt: Sansom, G.B., The Western world and Japan. A study in the interaction of European and Asiatic cultures, London 1950.

17 Zur Meiji-Restauration und den sie tragenden geistigen Kräften insgesamt: Beasley, W.G. (Hrsg.), The Meiji Restauration, Stanford 1972; Shiveley, Donald, Tradition and modernization in Japanese culture, Princeton 1971; Pittau, Joseph, Political thought in early Meiji Japan 1869-1883, Cambridge, Mass. 1967; Najita, Tetsuo, Japan. The intellectual foundations of Japanese politics, Chicago/London 1974. 
Den verschiedenartigen und zum Teil gegenläufigen Tendenzen der frühen Meiji-Zeit kam der Slogan "fukoku kyohei" (Bereicherung der Nation, Stärkung der Armee) am nächsten: Der gemeinsame Nenner der Modernisierungs- und Aufbruchsperiode lag in der selektiven Öfnung gegenüber ausländischen Einflüssen und Anregungen mit dem Zweck einer Stärkung des eigenen Volkes und seines Staates.

In der Balance zwischen konservativ-nationalistischen und westlich orientierten, aufklärerischen Kräften vollzog sich das geistige Ringen um den politischen Kurs des neuen Meiji-Staates. Am Ende konnte sich weder der extrem chauvinistische Nationalismus eines Motoori Nobinaga (1730-1801) noch die liberale, an rousseauistische Freiheitsvorstellungen anknüpfende "Bewegung für demokratische Freiheiten und Volksrechte" (jiyu minken undo) durchsetzen. ${ }^{18}$

Als bedeutsam erwies sich, daß Japan als erstes asiatisches Land eine konstitutionelle Ordnung erhielt. Die von Ito Hirobumi unter starkem Einfluß preußischer Verfassungsrechtler entstandene Meiji-Verfassung von $1889,{ }^{19}$ die Einführung eines Parlaments und in der Person General Yamagata Aritomos (1838-1922) eines Ministerpräsidenten, der Aufbau einer schlagkräftigen Armee nach zunächst französischen, dann - nach der Niederlage der Franzosen 1871 - preußischem Vorbild, die Einführung eines modernen Steuer- und Finanzsystems unter Zuhilfenahme britischer und amerikanischer Erfahrungen, die Reorganisation und Stärkung der Bürokratie und des Rechtswesens wiederum unter Anknüpfung an preußische Traditionen sowie eine umfangreiche Lokalverwaltungsgesetzgebung gehörten zu den tragenden Pfeilern der Meiji-Epoche und ihrer Modernisierungsmaßnahmen.

Entscheidendes Zeichen jener Jahrzehnte aber blieb die Reinstallierung des Kaisers, der durch die Verfassung von 1889 als geistliches wie politisches Oberhaupt der Nation definiert wurde, während der Shinto-Kult zur Staatsreligion erhoben wurde; oberstes Ziel war die Stärkung des "kokutai", des Volkskörpers.

Im Westen suchte Japan vor allem neues Wissen und Techniken der Gesellschaftsordnung, um die eigene Nation auf der Grundlage der traditionellen Wertvorstellungen zu kräftigen und ihr neue Perspektiven zu vermitteln. Japan trat in der Meiji-Zeit an, sich selbst zu modernisieren, um dem Westen an Stärke ebenbürtig gegenübertreten zu können. Zur vollen Reife gelangten die Intentionen der Meiji-Restauration, als die eigentlichen Traditionen des Landes postuliert werden konnten: in der späten Meiji-Zeit wurden konfuzianische Erziehungselemente - so in der "Imperial Rescription of Education" (1890) -, Loyalität, Autorität und die Anerkennung der hierarchischen Gesellschaftsordnung als Tugenden einer im Kern paternalistischen Staats- und Lebensstruktur propagiert.

Die Selbstentwicklung Japans fand ihre Grenze in der Berührung mit dem Menschenbild der westlichen Welt, dem Gedanken des Personalitätsprinzips und eines Individualis-

18 Hierzu: Bowen, Roger W., Rebellion and democracy in Meiji Japan. A study of commoners in the popular rights movement, Berkeley 1980.

19 Vgl. Siemes, Johannes, Helmut Roesler and the making of the Meiji state, Tokio/Rutland (Vermont) 1968. 
mus, wie er für die abendländisch-christliche Tradition konstitutiv ist. In der Bewahrung seines Gruppencharakters suchte Japan seine Stärke, um als künftige Weltmacht vom Westen Anerkennung zu erfahren.

Einen wesentlichen Beitrag zum Gelingen der Meiji-Restauration und vor allem der ihr folgenden ökonomisch-technologischen Modernisierung Japans leistete die hohe Aufmerksamkeit, die dem Bildungswesen gewidmet wurde. Die Grundlagen hierfür waren in den zweieinhalb Jahrhunderten der Tokugawa-Herrschaft gelegt worden. Zum Zeitpunkt der Meiji-Restauration besaßen 40 bis 50 Prozent aller japanischen Jungen und etwa 15 Prozent aller Mädchen eine formale Erziehung außerhalb des Elternhauses. ${ }^{20}$ Damit lag der Anteil der Alphabeten in Japan der zweiten Hälfte des 19. Jahrhunderts höher als in den meisten Ländern des Westens zu jenem Zeitpunkt.

Im späten 19. Jahrhundert wurde Japan von der ersten Industrialisierungswelle erfaßt. Die Grundlagen für den phänomenalen Aufstieg nach dem Zweiten Weltkrieg zu einer der führenden Industriemächte der Erde wurden schon in jener Zeit konsequent gelegt. Die großen Handelshäuser beispielsweise besaßen bereits eine mehrhundertjährige Tradition: So hatten die Gründer des Hauses Mitsui um 1620 als Sake-Brauer in der IseProvinz begonnen; 1690 war Mitsui zum ersten Finanzagenten für den damaligen Shogun und den Kaiserhof aufgestiegen. Sumitomos Wurzeln reichen ebenfalls in das 17. Jahrhundert zurück, als dieses Handelshaus im Drogen- und Eisenwarenhandel von Kyoto seinen Aufstieg begann; 1793 beteiligte Sumitomo sich führend an der Entwicklung der reichen Besshi-Kupferminen. ${ }^{21}$ Die Händlergemeinschaft der Tokugawa-Zeit hatte Japan bereits im 18. Jahrhundert in die Phase einer städtisch orientierten Handelswirtschaft geführt. Edo, das heutige Tokio, besaß schon damals eine Million Einwohner. ${ }^{22}$

\section{Auswirkungen}

Die Meiji-Restauration und ihre Konsolidierung wurden durch die erste Eruption der japanischen Militärmacht zu einem nationalen Klimax geführt. ${ }^{23}$ Der sino-japanische Krieg von 1894/95 endete mit dem japanischen Sieg und der Annexion Formosas. Die russische Seeniederlage von Tsushima reihte Japan 1905 endgültig in den Kreis der Mächte dieser Welt ein. 1910 wurde Korea annektiert und bis zum Ende des Zweiten Weltkrieges einer harschen japanischen Kolonialisierung unterworfen. ${ }^{24}$

20 Nach: Jansen, Marius (Hrsg.), Changing Japanese attitudes toward modernization, a.a.O., Seite 100.

21 Vgl. auch Piper, Annelotte, Japans Weg von der Feudalgesellschaft zum Industriestaat, Köln 1976.

22 Zur Stadtgeschichte in literarisch ansprechender Form: Seidensticker, Edward, Low city, high city. Tokyo from Edo to the Earthquake: how the Shogun's ancient capital became a great modern city, 1867-1923, Tokio/Rutland (Vermont) 1984 (4. Auflage).

23 Zum japanischen Nationalismus vgl.: Maruyama, Masao, Nationalismus in Japan. Historische Grundlagen und theoretische Perspektiven, in: Winkler, Heinrich August (Hrsg.), Nationalismus, Königstein/Taunus 1985 (2. Auflage), Seite $215 \mathrm{ff}$.

24 Vgl. Nish, Ian, Japan's foreign policy 1869-1942, London 1970; Morley, James William (Hrsg.), Japan's foreign policy 1868-1941, New York 1974. 
1899 hatte Japan die ihm 1856 aufgenötigten ungleichen Verträge mit den ausländischen Mächten revidieren können; 1902 wurde die englisch-japanische Allianz abgeschlossen. Erstmals konnte ein nichteuropäisches Land als gleichberechtigter Partner seine Unterschrift unter einen militärischen Allianzvertrag mit der damals führenden Macht setzen. Japan schien am Ziel seiner nationalen Wünsche angelangt.

Das 20. Jahrhundert aber war für Japan erfüllt von einer widersprüchlich erscheinenden Kombination von Planung und Irrationalität, von Berechnung und emotionalen Eruptionen, von Uberheblichkeits- und Minderwertigkeitskomplexen. Uber die Epoche der wechselnden Kabinette in den zwanziger Jahren der Taisho-Demokratie, die Militarisierung in den dreißiger Jahren - wobei der Aufstieg der Militärs zur führenden Kraft im Lande sich aufgrund ihrer Ambivalenzen im Rahmen der gegebenen Verfassungsstruktur vollzog und bei allen diktatorischen Ausdrucksformen ein eigentümliches Phänomen blieb, welches mit der europäischen Diktion des Faschismus keineswegs zu fassen und zu erklären ist -, der Ausbruch des militärischen Expansionismus zunächst gegen China und dann in voller Selbstüberschätzung gegen die Vereinigten Staaten, die Atombombenniederlage von 1945 und die Umwälzungen der Nachkriegsepoche künden auf vielfältige Weise davon. Bis zu den Diskussionen am Ende des Jahrhunderts um die japanische Exportpolitik und die Offnung japanischer Märkte für westliche Waren ist die verwirrende Widersprüchlichkeit nicht abgeklungen, die Japan stets umgab und in dieser Zuspitzung zu Unrecht den Mythos von der intellektuellen Undurchdringlichkeit jenes nationalstaatlichen Phänomens am Ostrand Asiens aufkommen ließ.25

Japan ist kein "westliches" Land geworden. Die Meiji-Restauration wurde bewußt vollzogen, um eine Zwangsverwestlichung abzuwehren und zugleich den Westen mit seinen eigenen Waffen zu schlagen. Die Niederlage Japans im Zweiten Weltkrieg, in welchem das Inselreich in einer hypertrophen Ubersteigerung den Angriff auf Pearl Harbour wagte und damit den Prozeß der schlußendlich eigenen Zerstörung einleitete, führte in vieler Hinsicht zu einer grundlegenden Neuordnung Japans.

Die Zerschlagung des Militarismus, die Neuordnung der Verfassungsverhältnisse - wobei dem Kaiser seine Stellung als oberste Shinto-Gottheit abgesprochen wurde und in Artikel 9 das japanische Volk auf sein souveränes Recht der Kriegsführung verzichtet -, die Landreform und eine Durchdemokratisierung der Gesellschaft in politisch-staatlicher Hinsicht haben zu einer Ordnung geführt, die in vielen Bereichen eine amerikanische Handschrift trägt und dem Land und seiner Bevölkerung eine zuvor ungeahnte Freiheit einbrachte.

Damit aber ist keine tatsächliche Verwestlichung Japans vollzogen. Bei aller formalen Einhaltung der Grundprinzipien parlamentarisch-repräsentativer Demokratie und ei-

25 Vgl. Nakane, Chie, Japanese society, Berkeley 1970; Courdy, Jean-Claude, Les Japonais, Paris 1979; Reischauer, Edwin O., The Japanese, Tokio/Rutland (Vermont) 1983 (29. Auflage); Wilkinson, Endymion, Japan versus Europe. A history of misunderstandings, Harmondsworth 1983; Barnet, Richard J., The Alliance. America-Europe-Japan. Makers of the postwar world, New York 1983; Maull, Hanns W., Europe and Japan: a relationship in search of roles, Auf zeichnung der Stiftung Wissenschaft und Politik, Ebenhausen 1985; zur Verfassungsneuordnung 1945: Röhl, Wilhelm, Die japanische Verfassung, Berlin 1963. 
nem beachtlichen Schutz der bürgerlichen Grundrechte weist das innenpolitische System Japans spezifische Kennzeichen auf, die mit der eigentlich westlichen Demokratieauffassung nicht deckungsgleich sind. ${ }^{26}$

Wohl mag mit dem Eintritt des "New Liberal Club" in die Regierung des Ministerpräsidenten Yasuhiro Nakasone im Dezember 1983 ein koalitionspolitisches Zeitalter eingeläutet worden sein; die Demokratischen Sozialisten und die Wohlfahrtspartei "Komeito" - der politische Sprößling der buddhistischen Sekte Sokka Gakai - rüsten sich zu einer möglichen späteren Teilhabe an der Macht und selbst die japanische Sozialistische Partei sah sich Mitte der achtziger Jahre genötigt, ihre starren Positionen in außen- und sicherheitspolitischen Fragen zu überdenken. Das prägende Element der japanischen Innenpolitik aber seit ihrer Gründung in den frühen fünfziger Jahren blieb bisher die Liberaldemokratische Partei (LDP), faktisch ein Zusammenschluß von fünf Flügeln im Sinne eines seit der frühen japanischen Geschichte bekannten politischen Faktionalismus. Die Gruppen innerhalb der konservativen Regierungspartei führen weithin ein Eigenleben; der Stil des politischen Lebens in Japan, die Tendenz zur Personalisierung und zur Loyalitätsverpflichtung innerhalb der eigenen Fraktion trägt spätfeudale Züge. Der personale, zuweilen fast lehenshaft anmutende Charakter der politischen Verhältnisse läßt zentrale Unterschiede zur westlichen Demokratieauffassung erkennen. Die Kombination des personalen Gefolgschaftsprinzips - im Sinne von "chu", dem klassischen Loyalitätsgedanken - und den aus dem Westen adaptierten Elementen des konstitutionellen Parlamentarismus scheint jedoch für Japan ein geeignetes Konzept zu sein, um die politischen Fragen der Nation zu bewältigen.

Der Gesamtbereich des Offentlichen, Politischen wird in der japanischen Tradition gern als "oyake", großes Haus, verstanden. Der Staat gilt als Wechselspiel und Beziehungsgeflecht von funktionalen Häusern (kokka). Das Konzept der Demokratie ist im politischen Denken Japans stets als zu abstrakt und zu normativ angesehen worden. Der japanischen Zielperspektive kommt die Verpflichtung auf den Harmoniegedanken weit näher als die demokratische Vorstellung der widerstreitenden Gruppeninteressen und Einzelforderungen.

Die intellektuellen Wurzeln der modernen japanischen Politik sind als konfligierende Interessen beschrieben worden: einerseits die bürokratische Tradition (kanryoshugi), die einem Effizienzdenken und einer "ethic of achievement "27 verpflichtet ist, andererseits der kulturelle Idealismus und Nationalismus, der mit einem enormen Grad an nationaler Selbstbezogenheit einhergeht. "Giri", das Gefühl der Verpflichtung - und "on" - das verpflichtende Antworten auf eine Wohltat - sind konstitutive Elemente dieser Mentalität geblieben. Aus ihrer Úberspannung lassen sich extreme Formen japanischen Nationalismus, die Attitüde, überall der Erste und Beste sein zu wollen und zu müssen, erklären, aber auch Abirrungen des Gehorsamkeitsprinzips, wie sie im Zweiten Weltkrieg durch die Kamikaze-Piloten versinnbildlicht wurden.

26 Vgl. Ike, Nobutake, Japanese politcs, New York 1972; spricht von einer "patron-client-democracy".

27 Najita, Tetsuo, Japan. The intellectual foundation of Japanese politics, a.a.O., Seite 5. 
Kaum irgendeiner Kultur der modernen Welt scheint ein derartiger Unterscheidungszwang zwischen dem eigenen Lebensgefüge und der Welt der Anderen zu eigen zu sein. Der Gruppencharakter der japanischen Gesellschaft tendiert zur strikten Abgrenzung gegenüber Außenstehenden. Die Soziologin Chie Nakane hat von "sotto" und "uchi" gesprochen, von "draußen" und "drinnen". ${ }^{28}$ Wer nicht dem "uchi", dem Haus, angehöre, steht außerhalb: von der Betriebsgruppe bis zur quasi-Großfamilie der Gesamtnation. Kritiker haben der japanischen Gesellschaft immer wieder einen Mangel an Religiösität, an spirituellen Interessen attestiert. In der Tat fehlt es an einer substanziellen japanischen Ethik oder Moralphilosophie. Anders als im religiös sehr empfänglichen Korea steht das japanische Volk der Religiösität eher indifferent gegenüber. Shinto-Tempelfeste und buddhistische Feierlichkeiten erwecken oftmals den Eindruck von Ritualhandlungen oder folkloristischen Kundgebungen. ${ }^{29} \mathrm{Da}$ viele Japaner sich zugleich als Shintoisten und Buddhisten bezeichnen, besagt für sich besehen noch wenig: Die tatsächlichen religiösen Kenntnisse und Empfindungen stehen oft in keinem rechten Verhältnis zur Tiefe der Glaubensinhalte selbst. Die westliche Religion des Christentums wurde nur von etwas mehr als einem Prozent der Japaner angenommen, ein scharfer Unterschied zum Nachbarstaat Korea, wo inzwischen fast ein Viertel der Bevölkerung protestantischen Kirchen oder dem römischen Katholizismus zugerechnet werden kann.

In einem sehr spezifisch japanischen Sinne aber besitzt auch das ostasiatische Inselvolk eine hochentwickelte Art der Religiösität und einen daraus abgeleiteten Moralkodex. Diese Religion mag man als "Japanismus" bezeichnen; ihr Moralkodex ist der "Weg des Kriegers" (bushido), der Pflichtenkatalog gemäß der Samurai-Sitten und das Rollenverhalten im Sinne einer Scham-Kultur. ${ }^{30}$

Wenngleich derartige anthropologischen Deutungen mit einer gewissen Vorsicht zu pauschalieren sind, nimmt es doch Züge des Religiösen an, in welcher Weise sich die Japaner quasi als eine Volksfamilie sehen und verstehen. Der Eintritt in den Japanismus vollzieht sich mit der Geburt, die Lebensstufen sind kollektiv festgelegt und werden jeweils kollektiv durchschritten.

Die nationalen Feiertage, an denen die drei-, fünf- und siebenjährigen Kinder zu den Shinto-Schreinen geführt werden oder an denen die im Vorjahr zwanzig Jahre alt gewordenen Jugendlichen gemeinschaftlich für volljährig erklärt werden, tragen archaisch-rituelle Züge und verblüffen im Kontrast zu der modernen japanischen Massengesellschaft im Zeitalter von Hochtechnologie und Wirtschaftsrationalismus. Der Shintoismus wird weiterhin als Ahnenkult verstanden und praktiziert. Darüberhinaus aber mutet

28 Nakane, Chie, Japanese. society, a.a.O.

29 Vgl. die Zusammenschau jener "matsuri“ genannten Festivitäten: Bauer, Helen/Carlquist, Sherwin, Japanese festivals, Tokio/Rutland (Vermont) 1983 (4. Auflage).

30 Vgl. die beiden noch immer klassischen Studien: Nitobe, Inazo, Bushido, The soul of Japan. An exposition of Japanese thought, Erstausgabe 1905, Tokio/Rutland (Vermont) 1983 (17. Auflage); Benedict, Ruth, The Chrysanthemum and the sword. Pattern of Japanese culture, Erstausgabe 1946, Tokio/Rutland (Vermont) 1983 (37. Auflage). Vgl. auch die sozialpsychologisch erhellende Studie: Doi, Takeo, The anatomy of dependence, Tokio 1973; deutsche Ausgabe: Amae - Freiheit in Geborgenheit. Zur Struktur japanischer Psyche, Frankfurt 1982. 
er - zumindest dem westlichen Beobachter - als eine ritualisierte Form der kollektiven Selbstanbetung eines ganzen Volkes an, wie es im von tiefen religiösen Traditionen erfüllten Asien einmalig ist. Im Shintoismus verehren sich die Japaner selbst.

Die ältesten überlieferten Texte der japanischen Literatur sind bezeichnenderweise jene, in denen die mythologische Abkunft der japanischen Inseln, des japanischen Volkes und des japanischen Kaiserhauses von der Sonnengöttin Amaterasu Omikami geschildert wird. Das "Nihongi" und das "Kojiki" nehmen in der geistigen Tradition Japans einen fast mit den Offenbarungsbüchern der westlichen Religionen vergleichbaren Platz ein.

Besonders auffällig im geistesgeschichtlichen Vergleich zwischen Japan und dem Westen bleibt die weitgehende Zurückweisung des christlichen Glaubens in Japan. Nur etwa ein Prozent der Bevölkerung von 120 Millionen Japanern bekennt sich zum Christentum. 1549 war mit Franciscus Xavier der erste ausländische Missionar in Kyushu gelandet. 1590 wurden nach einem Edikt des mächtigen Militärherrschers Hideyoshi alle Missionare des Landes verwiesen. Unterdessen waren mehrere Hunderttausend Japaner zum Christentum übergetreten. Die Verfolgung dieser Christen und besonders herausstechend die Hinrichtung von 26 einheimischen und ausländischen Christen 1596 in Nagasaki - heute erinnert daran eine Gedenkkirche in der Stadt des zweiten Atombombenabwurfs der Menschheitsgeschichte - gehört zu den dunkelsten Kapiteln der japanischen Geschichte jener Jahrhunderte der Berührung mit dem Westen. ${ }^{31}$

1614 wurde das Christentum endgültig in Japan verboten. Die niedergeschlagene Shimabara-Rebellion von 1637 - hinter ihr standen maßgeblich die im Untergrund treu zu ihrem Glauben haltenden Christen - forderte 37000 Tote. Die für über ein Jahrhundert fast vollständige Selbstisolation Japans nach 1639 führte zu einer weiteren Auszehrung des Christentums. Doch auch diejenigen, die treu zu ihrem Glauben standen, wurden in vieler Hinsicht von der sie umgebenden japanischen Lebensform und Kulturtradition förmlich aufgesaugt. ${ }^{32}$ Auch unter den Bedingungen demokratischer Freiheit nach 1945 vermochte sich das Christentum numerisch kaum beachtlich zu erweitern, wobei hingegen der soziale und kulturelle Einfluß der in Japan wirkenden christlichen Institutionen und ihre Auswirkungen auf die politische und persönliche Ethik überdurchschnittlich erkennbar wurde.

Dennoch sind es gerade immaterielle Fragen und Probleme im nichtökonomischen Bereich, in denen die bedeutsamsten Mängel der modernen japanischen Gesellschaft anzutreffen sind. Auch wenn die wirtschaftliche Struktur des Landes selbst durchaus fragile Züge trägt, so liegen die eigentlichen Schwächen, die hinter der Fassade imponierender technologischer Entwicklungen erkennbar geworden sind, doch gerade in jenen Bereichen, die ein höheres Maß an moralischem Wertbewußtsein, an civil sense und bürgerlicher Verantwortungsethik zu ihrer Úberwindung voraussetzen. Die ökonomischen "su-

31 Erneut sei auf das literarische Zeugnis jener Epoche der Christenverfolgungen verwiesen: Endo, Shusako, Silence (Chinmoku), Tokio 1983 (2. Auflage).

32 Endo sprach in dem zitierten Roman von einem "cultural mudswamp", der bezeichnend für Japan sei und jegliche geistige Anregung, die von außen in die Gesellschaft und ihre festgefügte Ordnung eindrang, stets aufgesaugt habe. 
per-achievers ${ }^{\prime 3}$ sehen sich immateriellen Wertdefiziten gegenüber, die auf alle modernen Massengesellschaften zutreffen mögen, in Japan stets aber auch die Identitätsgegensätze zwischen der eigenen Tradition und der westlichen Zivilisationsnorm aufscheinen lassen, wie sie seit den Tagen der Meiji-Restauration das Land geistesgeschichtlich durchdrungen haben.

\section{Japan und die Modernisierung der Dritten Welt}

Japan steht in der modernen Welt ohne Beispiel. Im ostasiatischen Vergleich ist Japan ökonomisch wie sozial führend, und im weltweiten Maßstab kann der Inselstaat neben den Vereinigten Staaten als das erfolgreichste Entwicklungsland der letzten Jahrhunderte angesehen werden. ${ }^{34}$

Die Begrenztheit der weltweiten Ressourcen, die enorme Kluft, die zwischen dem Massenwohlstand und der demokratischen Sozialstaatlichkeit in Europa, Nordamerika und Japan einerseits und den unterentwickelten Strukturen und sozialen Notlagen in der sogenannten Dritten Welt andererseits klafft, aber auch normative Uberlegungen zum bestmöglichen Entwicklungsmodell lassen heute viele Beobachter daran zweifeln, daß die Maßgaben der Industriezivilisation für die aufstrebenden Völker der Dritten Welt jemals erreichbar sein werden oder erreichbar sein sollten. Dennoch soll am Ende die Frage aufgeworfen werden, welche Rückschlüsse, Anregungen und Konsequenzen sich aus dem spezifisch japanischen Modernisierungsweg für die Länder der Dritten Welt ziehen lassen können. ${ }^{35}$

In Ostasien sind es vor allem Korea und Taiwan - beide indessen vorbelastet durch die Erfahrungen der japanischen Okkupation in der ersten Hälfte des sich zu Ende neigenden Jahrhunderts -, die nach Japan blicken und ökonomisch-technologischen Anschluß suchen. Aus der ASEAN-Gruppe ist es vorrangig Malaysia, das zumindest verbal seit den frühen achtziger Jahren eine "look east"-Politik begonnen hat. In entscheidender Hinsicht ist die Ausgangslage Japans ein Sonderphänomen und läßt sich nur schwerlich auf die Gegenwartslage der Dritten Welt übertragen. Japans lange, homogene Kulturtradition, seine Wurzeln im chinesischen Erbe, die frühzeitigen Bestrebungen von Handwerk und Handel, die rasche Industrialisierung am Ende des 19. Jahrhunderts, das Vermeiden einer kolonialen Eroberung, der eigenständige und eigenwillige Aufstieg zu einer imperialen und expansiven Großmacht, schließlich der totale Zusammenbruch nach der militärischen und diktatorischen Úberhitzung 1945 und der demokratische und marktwirtschaftliche Neubeginn finden kein Äquivalent in den meisten Ländern der heutigen

33 Asia-Week, Hongkong 20. April 1984.

34 Vgl. Kühnhardt, Ludger, Japan: the most successful developing country, in: The Ichigaya Times, No. 7, Summer 1984, Seite 4. (Tokyó-Sophia University).

35 Wir folgen bei unseren Betrachtungen keineswegs den zu extrem optimistischen Deutungen Hermann Kahns: Bald werden sie die ersten sein. Japan 2000. Zukunftsmodell der neuen Herren der Welt, Wien 1970. 
Dritten Welt; hier kann nicht schlechterdings nachvollzogen werden, was Japan seit der Mitte des 19. Jahrhunderts weitgehend aus eigener Kraft geleistet hat.

Viele der Fragestellungen, denen sich die Länder der Dritten Welt ausgesetzt sehen, finden im Vergleich mit dem japanischen Entwicklungsweg eine Erhellung und Klärung und verhelfen zur Selbstreflexion in jenen Staaten der Dritten Welt, über Asien hinaus auch in Afrika und Lateinamerika. Es sind vor allem drei Aspekte des japanischen Modernisierungsweges, die für die entwicklungspolitische Diskussion zum Nachdenken Anlaß geben können:

1. Während der Tokugawa-Zeit wurde größtes Augenmerk auf die landwirtschaftliche Entwicklung Japans gelegt. Die Insellage, die begrenzten Ressourcen und eine beständig wachsende Bevölkerung zwangen dazu, die Agrarproduktion und namentlich den Reisanbau zu steigern. Hungersnöte, Mißernten und Reisrevolten konnten nicht vermieden werden. Insgesamt jedoch war die agrarwirtschaftliche Entwicklung positiv. Die Uberschüsse aus dem Agrarbereich dienten frühzeitig dazu, den Handel auszubauen und im 19. Jahrhundert die ersten Grundsteine der Industrialisierung zu legen. Während in vielen Entwicklungsländern noch immer und irrtümlicherweise geglaubt wird, daß eine rasche Industrialisierung die Vernachlässigung der Landwirtschaft kompensieren könne, hat Japan frühzeitig und weitsichtig den logisch richtigen Weg eingeschlagen. Die Priorität der ländlichen Entwicklung und der Agrarproduktion bleibt für die meisten Entwicklungsländer am Ende des 20. Jahrhunderts die einzig überzeugende Antwort auf die Herausforderungen durch Bevölkerungsexplosion, Mangelwirtschaft, Devisenknappheit und Abhängigkeiten von der Natur und ihren Gewalten.

2. Die Meiji-Restauration wurde eingeleitet in dem Bewußtsein und vor dem Hintergrund eines hohen Bildungsstandards der breiten Bevölkerung. In der Tradition der konfuzianischen Ideale und der Wissenschaften Chinas hatte Japan frühzeitig ein formales Bildungssystem aufgebaut, in welchem die klassischen Bücher Chinas gelehrt und das eigene Verwaltungspotential herangebildet wurden. Auf der Basis einer für die damaligen Verhältnisse im Weltmaßstab erstaunlich hohen Alphabetisierung und einer qualifizierten, den nationalen Interessen verpflichteten Verwaltung (kanryoshugi) nahm Japan den Wettbewerb mit seiner eigenen Rückständigkeit und der westlichen Herausforderung optimistisch und selbstbewußt auf. Das japanische Beispiel verdeutlicht gegenüber den Entwicklungsländern der Welt von heute den engen Zusammenhang zwischen Bildungsanstrengungen für die breiten Schichten, dem Aufbau einer qualifizierten und den nationalen Zielen verpflichteten Verwaltung und einer insgesamt erfolgversprechenden Modernisierung einer Gesellschaft.

3. Auf ihre je unterschiedliche Weise traten die Vereinigten Staaten von Amerika und Japan mit einer nationalen Idee oder gar Ideologie an, um sich von Europa zu emanzipieren und von den Europäern als gleichwertiger Partner respektiert zu werden, eigenständiges Gewicht in einer sich wandelnden Welt zu erlangen und die nationalen Entwicklungsdefizite auszugleichen. Lag für die Vereinigten Staaten das sinnstiftende Element in der liberaldemokratischen Verfassungsgebung - all men are born equal -, so galt für Japan die Stärkung des "kokutai", des Volkskörpers als Antrieb der Modernisie- 
rungsepoche. Interessanterweise stand in beiden Fällen nicht eine primär ökonomisch oder sozial ausgerichtete Konzeption, sondern ein politisch-geistiges Programm am Beginn eines nationalen Erfolgsweges, der wie selbstverständlich auch die wirtschaftlichen und sozialen Belange und Anliegen der beiden Völker umschloß und erfüllte.

Vielen Entwicklungsländern mangelt es heute gerade an dieser längerfristig ausgerichteten geistig-politischen Perspektive. Die unreflektierte Uberstülpung des spezifisch europäischen Nationalstaatskonzeptes kann darüber ebensowenig hinwegtäuschen wie die oftmals zur nationalen Ideologie erhobene entwicklungspolitische Aufgabe, in deren Namen viele Staaten und ihre Eliten die künstliche Alternative wirtschaftliches Wachstum versus politische Freiheit aufkommen ließen. Hunger und Unterentwicklung aber lassen sich dauerhaft weder ohne politische Freiheit und Mitgestaltung noch ohne eine Beantwortung der gesamtgesellschaftlichen Zukunftsorientierung überwinden.

Japans Stärke liegt - neben anderen Aspekten - in dem eigentümlichen Rationalitätsprinzip begründet, welches dieses asiatische Volk leitet. Effizienz, Effektivität, Erfolgsorientierung, Streben nach Verbesserung des Bestehenden, der Hang gar zur permanenten Perfektionierung und ein alles durchflutender Bildungshunger paaren sich mit einem nachgerade archaischen System gesellschaftlicher Regeln und Regulierungen, Etiketten und Normen, die of t kunstvoll, aber doch in einer die individuelle Persönlichkeitsentfaltung hemmenden Weise Volk und Gesellschaft zusammenschweißen. Die gesellschaftlichen Grundgefühle Japans und der westlichen Welt liegen in vieler Hinsicht weit auseinander. In einer interdependenten Welt aber muß die geistesgeschichtliche Dimension ebenso berücksichtigt werden wie Fragen der wirtschaftlich-technologischen Kooperation. In ganz besonderer Weise gilt dieses für das dem Westen noch immer verwirrend fremd und mystisch erscheinende Japan, jenes gleichzeitig extrem konservative und moderne, traditionsverhaftete und zukunftsgewisse Land am Ostrand der eurasiatischen Landmasse. 


\section{Japan: The Conservative Revolution}

\section{By Ludger Kühnhardt}

The roots of modern Japanese society have been established during the 19th century. The Meiji-Era has been considered as one of the most interesting historical transformations in world history. The Japanese started to modernize their society along the lines of Western methods and techniques while rejecting the very foundations of Western civilization, Christian religion and the individualistic philosophies of the Age of Enlightenment in particular. After 250 years of Shogunal rule, the Meji-Restoration provided for the shift in political power in favour of the imperial régime. 1868 became the turning point of Japanese history. The remarkable historical development which followed transformed Japan into one of the leading world powers. It would, however, be misleading to describe Japan as a Westernized country. The opposite is true: notwithstanding the Meji-Era and the fundamental changes in the social, political and cultural structure of the country and its society after the Second World War, Japan remains basically an Oriental society, almost anti-Western with respect to the foundations of its intellectual fabric and notions of the individual. The article describes the historical and intellectual development of Japan with particular emphasis on the Meiji period which ended with the promulgation of the Meiji constitution in 1889: it provided Japan as the first Asian country with a modern constitutional system.

\section{The Philippines: An Anatomy of the Rise and Fall of a Dictatorship}

\section{By Rainer Werning}

This paper describes the reign of the former Philippine dictator Ferdinand E. Marcos, the political aims and interest groups guiding his régime, and the emergence of the succeeding administration led by Corazon Aquino.

Intricate internal coalitions as well as foreign involvement, especially of the United States of America, continue to subtend the new Aquino government while the radical opposition to the Marcos oligarchy has been largely bypassed by the post-Marcos political dispensation. 